\title{
Experimental Assessment of Zigbee As The COMMUNICATION TECHNOLOGY OF A WIRELESS SENSOR NETWORK FOR GREENHOUSE MONITORING
}

\author{
Ilias Lamprinos ${ }^{1}$ and Marios Charalambides ${ }^{1}$ \\ ${ }^{1}$ Telco Software Department, Intracom Telecom, 19.7 km Markopoulou Ave, \\ 19002, Peania, Greece
}

\begin{abstract}
Wireless Sensor Networks are considered an important part of the modern ICT solutions for greenhouse monitoring. Several communication technologies are already available and have been used both in pilot and commercial installations. Their attractiveness is based on their deployment flexibility and low cost. However, some special features of the greenhouses and their cultivations (variable microclimate conditions, greenhouse construction materials, size and composition of plants, spatiotemporally variable foliage) raise questions about the proper topology and network density in such an environment. In this paper, the findings of our experimentation regarding the use of ZigBee as the communication technology for a WSN deployed in a greenhouse complex with hydroponic cultivation of tomato crops are presented. Different topologies were investigated and the impact of the plants' foliage in the network nodes connectivity is assessed.
\end{abstract}

\section{Keywords}

Greenhouse Monitoring, Wireless Sensor Networks, Environmental Sensing, ZigBee

\section{INTRODUCTION}

In recent years several factors have led to an increase of agriculture production under controlled environments. For this type of production, greenhouse cultivations constitute the most productive form. Today, greenhouse industry in Europe is a leading industry in horticulture with more than 200,000ha covered by greenhouses and 200,000 active enterprises, employing 700,000 workers and having a turnover of about $€ 80$ billion [1].

After labour expenses, energy is typically the largest overhead cost in the production of greenhouse crops in temperate climates. Of the total energy consumed, roughly $65 \%$ to $85 \%$ is for heating, and the remainder is for electricity and transportation. In an industry with declining profit 
margins and an increased desire for sustainability, greater emphasis is being placed on producing greenhouse crops in an energy-efficient and environment-friendly manner.

Among the key factors that affect the quality and productivity in a greenhouse there are several environmental conditions indicators, such as temperature, humidity, light and the carbon dioxide level. Continuous monitoring of these parameters enables the grower to better understand how each of them affects crops' growth and proper control of them can result in higher production efficiency and quality [2].

Towards this direction, the role of modern ICT is fundamental, and one of the key enabling technologies is the Wireless Sensor Networks (WSN). A WSN consisting of small-sized sensor nodes equipped with radio and one or several sensors is a basic part of the modern greenhouse monitoring systems. On the basis of the information collected by the sensor nodes in a central station, an intelligent software application could take decisions that will eventually be fed as set points to actuator nodes that perform the appropriate control actions. Such an approach would allow users to sense and control the greenhouse cultivation environment from anywhere [3].

In fact, the mapping of climate/crop spatial variability provides a way to adjust production inputs in different greenhouse compartments according to the needs of individual areas (e.g. greenhouse compartments having different crops, or the same crop at different phenological stages) rather than applying uniform set-points of water, energy and fertilisers applications across the entire greenhouse without accounting for climate and crop variability.

Apparently the successful introduction of WSN in horticulture needs the input of background knowledge from both scientists and horticultural engineers. In the context of the research project PEISMON novel technologies are developed and piloted that facilitate greenhouses' environmental monitoring with the use of a WSN. The project aims to meet the above- mentioned engineering and scientific challenges in a synergetic way.

The current trend of greenhouse crop management towards a tighter control of inputs and outputs (i.e. aimed at balancing resource inputs with crop demand) implies the need for:

(i) Characterising intra-greenhouse climate distribution; and

(ii) Enhanced analysis, interpretation and valorisation of the collected data.

Typically, several measurement points are required to monitor the greenhouse's microclimate parameters and ensure optimal climate, irrigation and fertilization management. Towards the accomplishment of this need in our early experimentation we deployed a WSN following the architecture presented in Figure 1. It consists of several environmental sensor nodes deployed in adjacent greenhouse compartments that periodically communicate their measurements to a central monitoring and control station, placed in a control room. Part of the WSN is also a weather station located in the open air, close to the greenhouse compartments. 


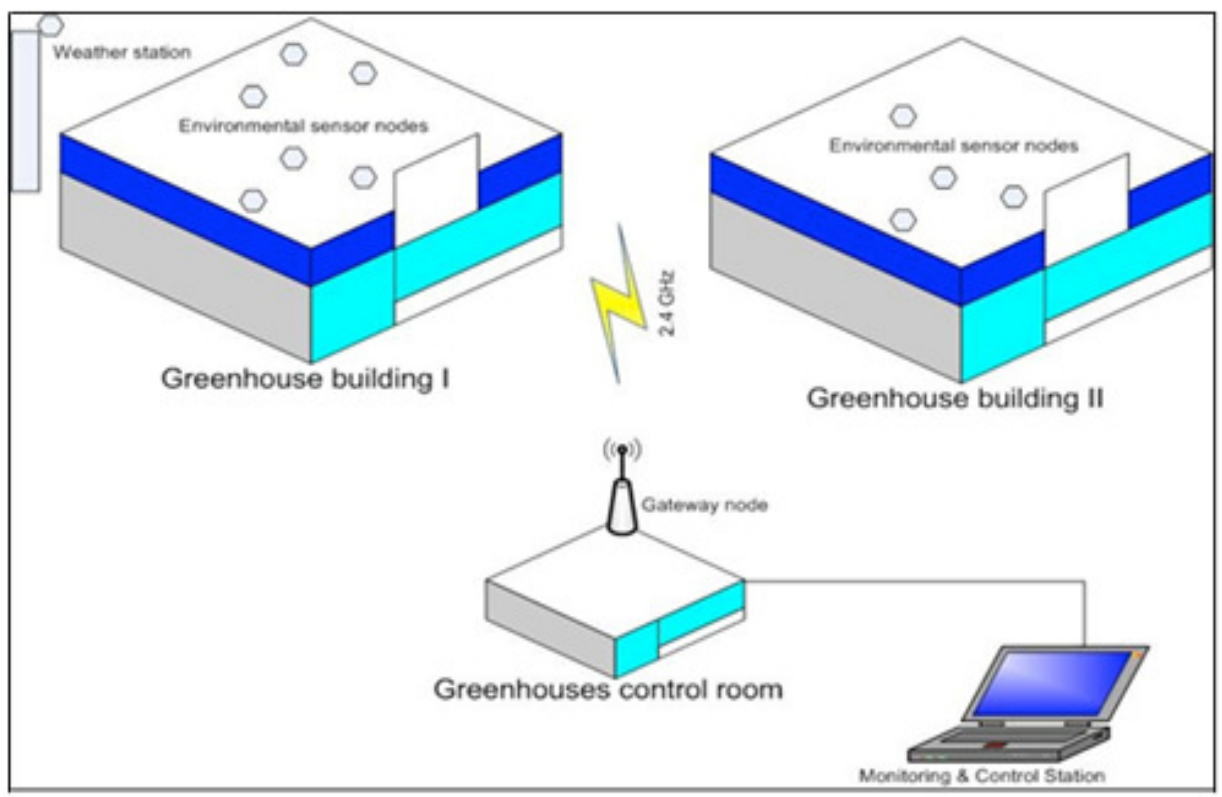

Figure 1. High-level system architecture

One of the issues that our research team investigated at the early stage of the project was the selection and experimental assessment of the communication technology used in the WSN. As a first decision, we only considered wireless technologies due to their deployment flexibility and low cost. There are a few types of such technologies that operate at similar, license-free frequency bands (e.g. ZigBee, Wi-Fi and Bluetooth). When considering factors such as supported data rate, number of nodes, energy consumption, nominal maximum distance among nodes, set up cost, installation complexity, and overall reliability, then ZigBee is reported to comprise the best solution [4]. The advantages of using ZigBee are mainly the fact that it is easy to use at the application layer, that is characterized by self-organization, that it has low power consumption, and finally that the typical cost of a ZigBee chip is low [5].

Before the final decision of adopting ZigBee as the communication technology for our system, we executed a set of experiments, triggered by reports of collaborating researchers (agronomists) about a series of specific problems related to the use of WSN in greenhouse environments. Such issues are:

- The impact of the foliage of the growing up plants: The appearance of the foliage medium in the path of the communication link has found to play a significant role on the quality of service (QoS) for wireless communications over many years [6]; 
- The impact of the size and the composition of the greenhouse plants: In the frequency of 2.4 $\mathrm{GHz}$ used in many WSN communication technologies, the signal attenuation due to water rich objects such as the plants is significant [7]. Also, the signal attenuation has a linear relationship with the volume of the vegetation [8]; and

- The impact of the greenhouse building materials: Due to the large amounts of metal parts in the greenhouse structure the electromagnetic field is completely different to that of an open space, and this can affect the quality of communication among the nodes of the WSN.

\section{RELATED WORK}

A set of special conditions within greenhouses (variable microclimate conditions, greenhouse construction materials, size and composition of plants, spatiotemporally variable foliage)raise some questions about the proper operating frequency, the topology and the network density of the WSNs that are used in modern greenhouse monitoring and operation control systems. Specifically for WSNs that operate in the license free $2.4 \mathrm{GHz}$ band it is reported that they are severely affected by the specific conditions inside the greenhouse during the crop growth period [5]. If the type of crop being grown is tall and produces large volumes of green mass, then not only the communication range is dramatically reduced, but it also varies by orders of magnitude within the crop growth cycle. It was measured that the initial range of a few dozen meters might drop down as drastically as two orders of magnitude, to 1-2 meters in case of tall crops like for instance paprika [9]. Salleh et al presented a system to monitor the greenhouse temperature and humidity parameters by applying ZigBee as the WSN communication technology [10]. In their range tests they found that the ZigBee receiver could get data from the transmitter even at a distance of $100 \mathrm{~m}$ but those tests were executed in the open field where the line of sight occurred. They also found that the reception was very slow in the distance range between 60 meters to 100 meters. However, when testing their system in a real greenhouse environment, with variable temperature and humidity conditions, the distance between the communicating nodes was limited to 22 meters. Quan et al experimented with a Digi ZigBee wireless module and found out that for indoor/urban area and for a range of up to $60 \mathrm{~m}$, the transmitted packets were properly received, however for distances larger than $60 \mathrm{~m}$ the signal strength dropped significantly and the number of packets was reduced by half at $65 \mathrm{~m}$ and totally at $75 \mathrm{~m}$ [11]. It is worth to note that the performance indicated in this work is related to indoor/urban conditions, but no special attention was paid to greenhouse specific conditions.

\section{Materials \& Methods}

\subsection{WSN monitoring application}

The Wireless Sensors Network is part of the infrastructure of a Greenhouse Monitoring and Management software. This is a suite of applications that enable the growers / operators to keep track of the cultivation activities that are carried out in the greenhouse environment. The suite includes the following two applications:

Cultivation Electronic Record (CER): This application covers aspects related to the monitoring, management and logging of the cultivation processes in correlation with the use of pesticides and the overall greenhouse environmental conditions. The application runs in an application server hosted by either the Greenhouse Operator himself or a third party Service 
Provider (i.e. software-as-a-service), and is accessible via web browsers running in standard PCs or tablets / smartphones. This application presents also the information collected by the WSN and stored in the central database. It keeps track of spatiotemporal information related to the whole cultivation cycle and serves as an Electronic Record for the products of the greenhouse. Figure 2 illustrates an indicative screenshot of the web application.

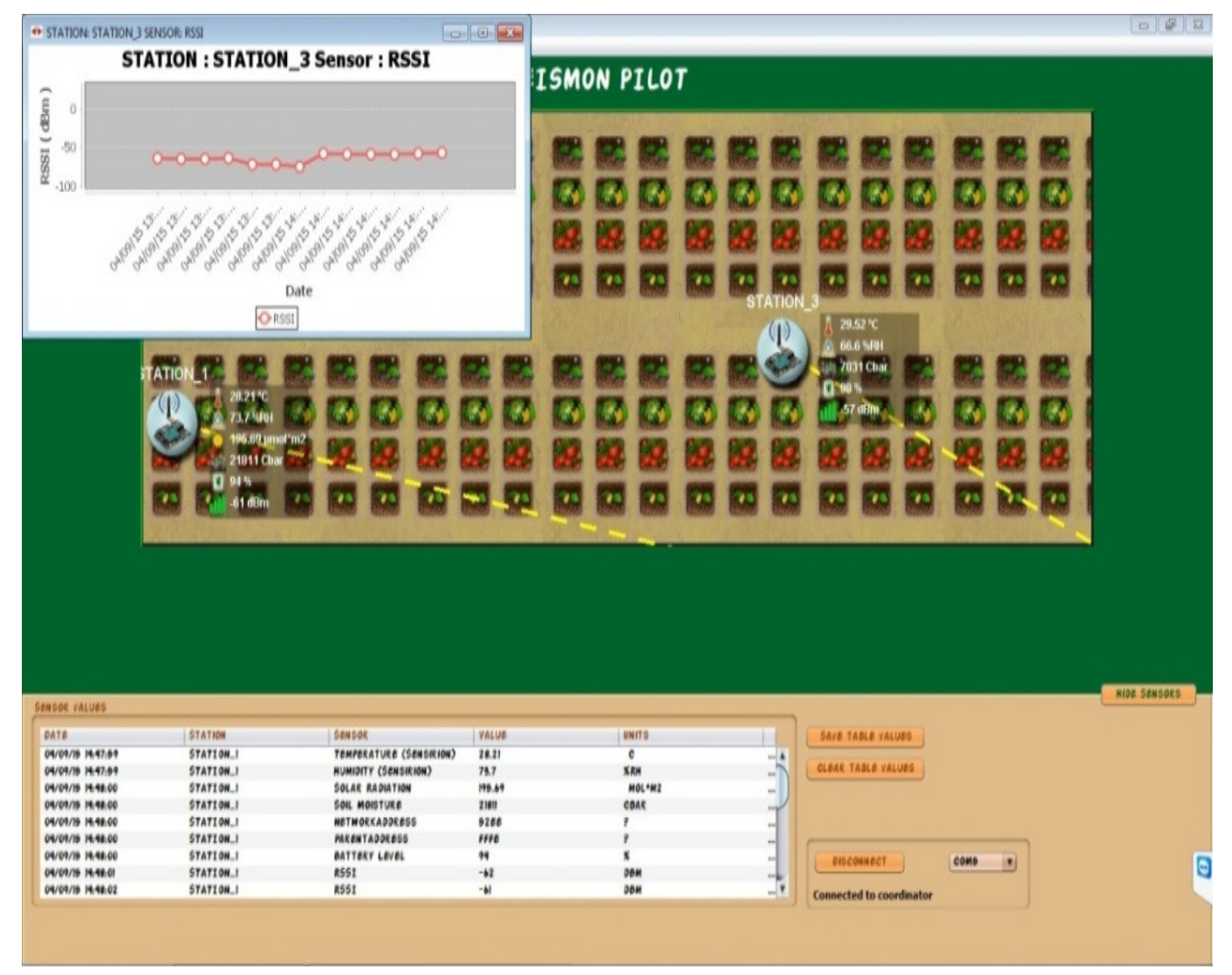

Figure 2. Measurements reception and presentation in the WSN monitoring application

WSN Monitoring and Control application: This is a desktop application that is responsible for collecting and handling locally the measurements of the WSN. Typically this application is hosted by a machine that is physically installed in the greenhouse's environment. Its role is twofold: first it enables the set up and management of the WSN nodes; and second it presents locally the collected measurements and stores them in the remote database, making use of appropriate web services that run at the application server of the CER. The WSN Monitoring and Control application covers visualization aspects related to the network topology, the nodes' status, the sensed data, but also the post processing information related to the monitored phenomenon. Figure 3 shows a screenshot of the desktop application. 


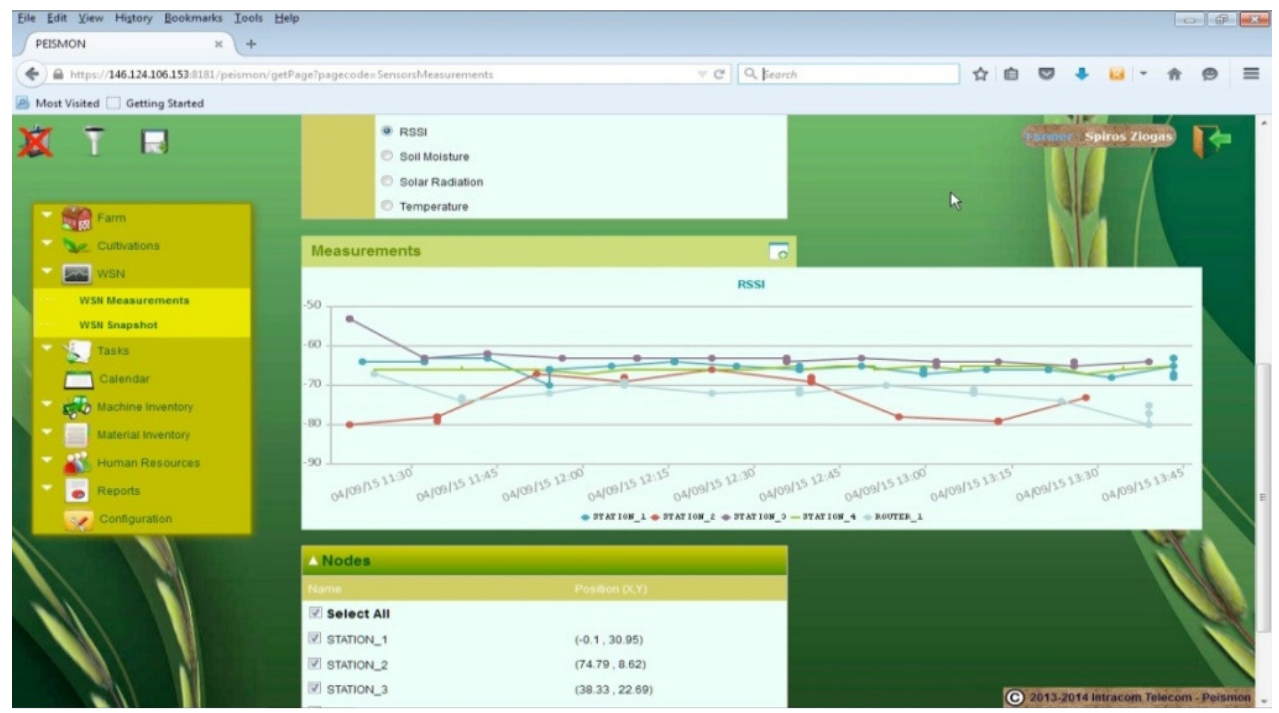

Figure 3. Presentation of the WSN measurements in a web based Cultivation Electronic Record application

\subsection{WSN topology in an experimental setup}

To realise the networking capabilities of the sensor nodes of a ZigBee WSN a set of experiments were conducted in a greenhouse environment. The main objective was to verify the statements of the vendor about their coverage and assess the impact of the greenhouses' special conditions in the typical distance among the communicating nodes. Another objective was to assess the level of autonomy of the sensor nodes when using PV panels as power sources, placed under the plastic sheet that surrounds the greenhouse.

The experiments were conducted in a complex of three greenhouse compartments and an adjacent utility room, as depicted in Figure 1. The greenhouse compartments were made of a metal frame and plastic sheets of polyethylene film (typical greenhouse construction in the Mediterranean area) and each was covering an area of $160 \mathrm{~m} 2$. Compartments \#1 and \#2 included hydroponic cultivation of tomato, whereas compartment \#3 had no growing plants at the phase of the experiments. The WSN monitoring application was running in a Personal Computer placed in the utility room (the Microclimate Monitoring Centre) and was communicating with the sensor nodes, via a gateway installed in the ceiling of the building, in the nearest corner to the greenhouses complex.

\subsection{Sensor nodes \& sensors}

The objectives of the experimentation were to investigate the performance of different topologies in terms of communication coverage. The communication technology was based on ZigBee and the deployed network included the following set of nodes:

- Six end nodes:three of them (indicated as Sensor Board 1,3,5 in Figure 2)were plugged in a Waspmote Agriculture Sensor Board v2.0 that included a temperature and humidity sensor(SHT75), a soil temperature sensor (PT-1000), and a solar radiation sensor (SQ 110); the other three (indicated as Sensor Board 2,4,6 in Figure 4) were plugged in a 
Waspmote Gas Sensor Board v2.0 that included a temperature sensor (MCP9700A), a humidity sensor (808H5V5), and a carbon dioxide (CO2) sensor (TGS4161);

- The central Coordinator (gateway) node; and

- Two router nodes (indicated as Router Board 1,2 in Figure 4).

All these nodes had as a radio interface an XBee PRO S2 ZigBee module with maximum transmission power of $50 \mathrm{~mW}$, sensitivity of $-102 \mathrm{dBm}$ and theoretical range of $7 \mathrm{~km}$ in the line of sight with a Fresnel zone clearance and $5 \mathrm{dBi}$ dipole antenna [12]. The nodes were programmed to operate as follows:

- Coordinator: It selects a channel and PAN ID to start the network; it allows routers and End Nodes to join the network; it is always on; it buffers data packets for sleeping end nodes

- Router: It must join a PAN before it can transmit, receive, or route data; after joining a PAN, it allows other routers and End Nodes to join the same network and assists them in routing data; it is always on and it can buffer data packets for sleeping end nodes;

- End Node: It must first join a PAN before it can transmit or receive data; it cannot allow devices to join the network; it cannot route data; it can enter low power modes to conserve power and can be battery-powered.

The End Nodes were programmed to get measurements every minute and scan for a parent node (either the Coordinator or a Router Node) to transmit their data. If more than a parent nodes were present in their scanning the End Nodes were programmed to select the one with higher Received Signal Strength Indicator (RSSI) due to connection reliability issues.

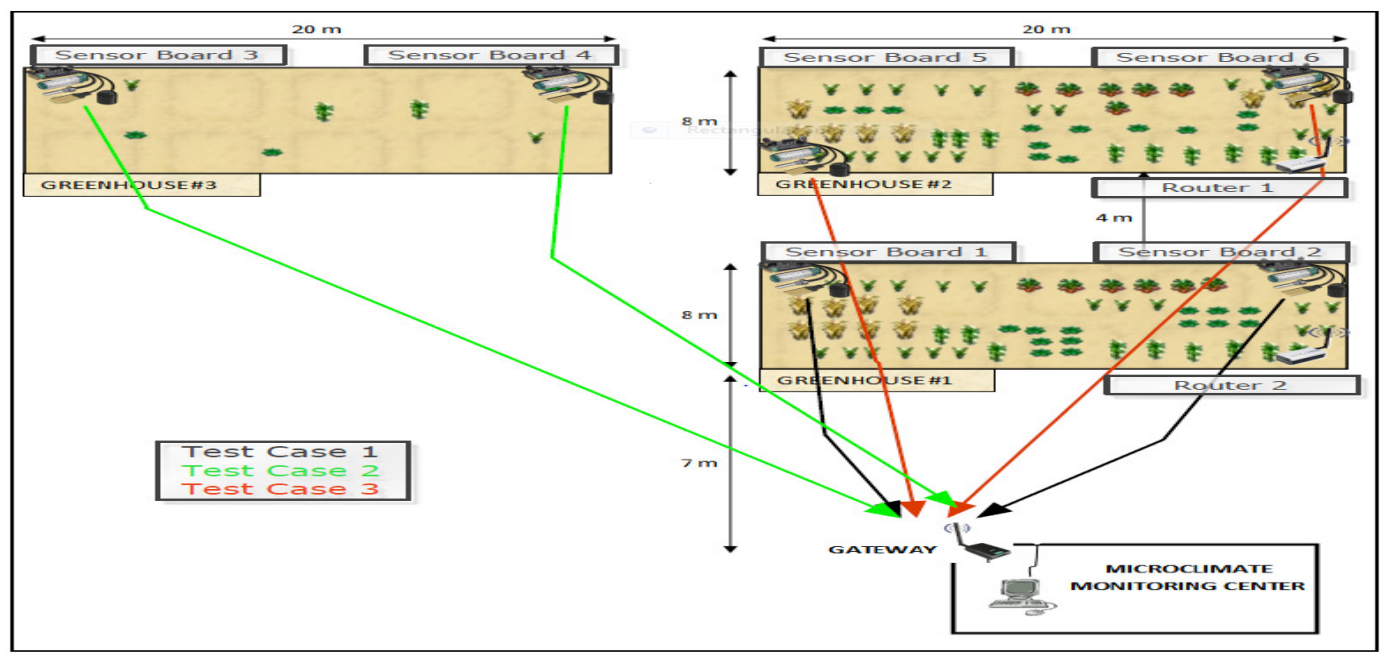

Figure 4. Sketch of the greenhouse layout where the experiments took place

\section{RESULTS}

We investigated three different topologies and the corresponding test cases (Figure 4). 
In the first test case two End Nodes were installed in compartment \#1, which included hydroponic tomato crop cultivation. The nodes were hanging in a high of 1.5 above the ground level and their distance from the Coordinator was $\sim 15-18 \mathrm{~m}$. All the attempts to communicate their measurements to the Coordinator were successful, and the average RSSI value was $-13 \mathrm{dBm}$. Overall, in this test case, the connectivity was continuous and the two End Nodes did not need routers to connect to the Coordinator.

In the second test case two End Nodes were installed in compartment \#3, which did not include any cultivation. The nodes were hanging in a high of 1.5 above the ground level and their distance from the Coordinator was $\sim 50 \mathrm{~m}$. All the attempts to communicate their measurements to the Coordinator were successful and the average RSSI value was $-17 \mathrm{dBm}$. So, also for this test case, direct connectivity between the End Nodes and the Coordinator was possible.

In the third test case two End Nodes were installed in compartment \#2, which included hydroponic tomato crop cultivation. The nodes were hanging in a high of 1.5 above the ground level and their distance from the Coordinator was $\sim 20-30 \mathrm{~m}$. Also, the cultivation of compartment \#1 was interposed between these nodes and the Coordinator. The Sensor Board 5 could connect to the Coordinator, while the Sensor Board 6 could not. Then, we switched on Router_1 and Router_2 that were placed close to the wall of compartments \#2 and \#1, as indicated in Figure 2. The Sensor Board 6 could now communicate its measurements to the Coordinator via Router_1. Also, the Sensor Board 5 selected to transmit its data via Router_1, due to better signal strength compared to that of the Coordinator.

With regards to the autonomy of the sensor nodes, we programmed four sensor nodes to communicate their measurements (humidity, temperature, solar radiation, and battery level), to the gateway once per ten minutes. The experiment took place during summer period. The battery level information collected after 40 days of experimentation is presented in Figure 5. The collected information indicates that there is no significant impact of the plastic sheet that surrounds the greenhouse in the charging process of the PV panels that source the battery of the sensor nodes, and therefore that the sensor nodes can operate in autonomous mode for significantly extended periods of time.

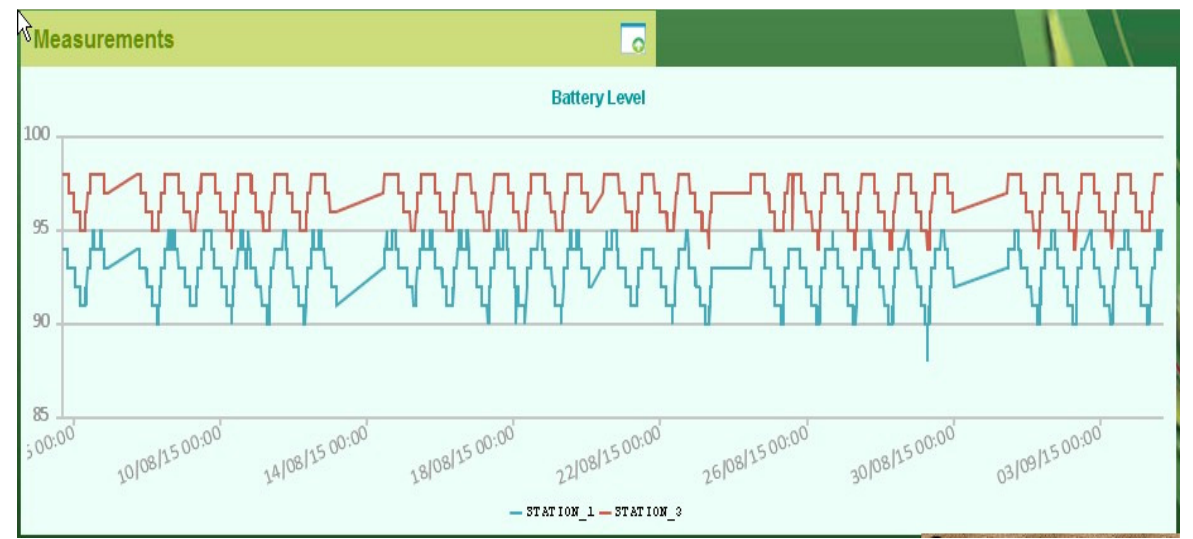

Figure 5. Battery level of a set of sensor nodes communicating with the gateway once per ten minutes 


\section{DISCUSSION}

Our experimentation was set up in a greenhouse complex with various characteristics in terms of foliage in order to assess its impact in the propagation of the radio waves. The results indicate that the lightweight construction of the greenhouse with plastic sheets over a metal frame did not have significant impact in the communication among the WSN nodes. However, the presence of plants and their foliage did contribute in the attenuation of the transmitted radio signal strength. In contrast to the nominal coverage distance indicated by the vendor (several hundreds of meters) the actual distance between the communicating nodes in a real greenhouse environment with tomato crops was rather limited (less than 30 meters). This indicates that non- deterministic obstacles affect the quality of communication among the nodes and impose the need for the deployment of a tree topology in which router nodes are introduced in the greenhouse field. The more foliage interposed in the propagation path between the network's nodes, the higher should be the density of the router nodes in order to guarantee the communication with the coordinating node. Actually, the spatiotemporal variability of the foliage should be carefully considered along with the variable microclimate conditions (not examined in the experimentation presented in this paper) in order to deploy a WSN that not only is well functioning, but also energy efficient.

\section{Conclusions}

This paper addresses an important issue related to greenhouse monitoring: the performance of ZigBee as the communication technology of the WSN that comprise the modern greenhouse monitoring and control systems. The experimentation with this technology in a real greenhouse environment with growing up plants revealed that the impact of the foliage of the cultivated crop significantly affects the performance of the network in terms of distance among the communicating nodes. Therefore, one important issue for consideration by the ICT system integrators of greenhouse monitoring solutions is that of the network topology and density. The nominal distance values provided by the WSN vendors should not be misconceived and extendedexperimentation is necessary to achieve a well functioning deployment of the WSN within a greenhouse, notwithstanding the energy efficiency aspects of the network operation that should be taken into consideration.

\section{ACKNOWLEDGEMENTS}

The PEISMON project received co-funding from the Greek General Secretariat for Research \& Technology. The authors would like to thank their colleagues in PEISMON project for their collaboration.

\section{REFERENCES}

[1] Eurostat, European Commission, 2010

[2] Timmerman, G. J., Kamp P. G. H., 2002. Computerised Environmental Control in Greenhouses. PTC, The Netherlands, 2nd edition.

[3] Gonda L., Cugnasca C.E., 2006. A proposal of greenhouse control using wireless sensor networks. Proceedings of 4thWorld Congress Conference on Computers in Agriculture and Natural Resources; Orlando, Florida, USA 
International Journal of Advanced Smart Sensor Network Systems (IJASSN), Vol 5, No.3/4, October 2015

[4] Ling-ling Li, Shi-Feng Yang, Li-Yan Wang, and Xiang-Ming Gao, The Greenhouse Environment Monitoring System Based on Wireless Sensor Network Technology, IEEE International Conference on Cyber Technology in Automation, Control, and Intelligent Systems, March 2011

[5] K Berezowski, B Wojciechowski, K Berezowski, P Patronik, J Biernat, The Landscape of Wireless Sensing in Greenhouse Monitoring and Control, International Journal of Wireless \& Mobile Networks (IJWMN) 4 (4), 141-154

[6] Y. S. Meng and Y. H. Lee, "Investigations of foliage effect on modern wireless communication systems: a review," Progress In Electromagnetics Research, Vol. 105, 313-332, 2010

[7] Tuijl, B. van; Os, E. van; Henten, E. van. Wireless Sensor Networks: State Of The Art And Future Perspective. Acta Horticulturae, Wageningen, 801. v.1, p. 547 - 554, 2008

[8] J. Giacomin and F. Vasconcelos, "Wireless sensor network as a measurement tool in precision agriculture," In Proc. XVIII IMEKO World Congress - Metrology for a Sustainable Development, Rio de Janeiro, Brazil, September 2006

[9] J. Hwang, C. Shin, and H. Yoe. A wireless sensor network-based ubiquitous paprika growth management system. Sensors, 10(12): 11566-11589, Jan. 2010

[10] Salleh A, Ismai M K, Mohamad N R, Abd Aziz M Z A, Othman M A, Misran M H. Development of Greenhouse Monitoring using Wireless Sensor Network through ZigBee Technology. International Journal of Engineering Science Invention, 2013; (2): 6-12

[11] Quan Minh Vu, Automated Greenhouse Management System, Master Thesis, School of Engineering and Advanced Technology, Massey University, June 2011

[12] Libelium Comunicaciones Distribuidas S.L., Waspmote Technical Guide, p26, 2013 